\title{
IMPORTANCE OF MAGNETIC EFFECTS IN A TWO-FLOW MODEL FOR EXTRAGALACTIC RADIO JETS
}

\author{
H. SOL ${ }^{1}$, G. PELLETIER ${ }^{2}$, E. ASSEO ${ }^{3}$ \\ ${ }^{1}$ DARC, Observatoire de Paris, 92195 Meudon Cedex, France \\ ${ }^{2}$ Groupe d'Astrophysique, Université de Grenoble, CERMO, BP 68, \\ 38402 Saint Martin d'Hères Cedex, France \\ ${ }^{3}$ Centre de Physique Théorique, Ecole Polytechnique, 91128 \\ Palaiseau Cedex, France
}

We propose a model for extragalactic radio jets in which two different flows of particles are taken into account, (i) a beam of relativistic electrons and positrons extracted from the funnel of accretion disc and responsible for the observed superluminal motion, (ii) a classical or mildly relativistic wind of electrons and protons coming out from all parts of the disc (Sol et al., 1989). Studying the mutual interaction of the two flows, we show that the configuration is not destroyed by the plasmabeam instability as long as the magnetic field, assumed longitudinal, is strong enough, with an electron gyrofrequency $\omega_{c}=e B / m_{e} c$ greater than the ambient plasma frequency $\omega_{p}=\left(4 \pi n_{p} e^{2}\right)^{1 / 2}$ (Pelletier et al., 1988). When $\omega_{c}<\omega_{p}$, the relativistic beam loses its energy and its momentum mainly through the development of strong Langmuir turbulence in the wind, and disappears quietly after some relaxation zone where heating and entrainment of the wind occur. This emphasizes one aspect of the important role likely played by the magnetic field in the dynamics of extragalactic jets and provides one example in which the magnetic field, acting on the microscopic scale of an interaction, induces strong effects on large-scale structures. Detailed data on the closest known superluminal radio source $3 \mathrm{C} 120$ (Walker et al., 1987, 1988; Benson et al., 1988) allow a check on the likelihood of our model. Observational estimates of the variation along the jet of the magnetic field and of the ambient plasma density $n_{p}$ suggest that the magnetic field reaches its critical value $B_{c}=3.2 \times 10^{-3} \sqrt{n_{p}}$ (corresponding to $\omega_{c}=\omega_{p}$ ) at a minimal distance of about $1.4 \mathrm{kpc}$ from the central engine. This is amazingly close to the location of the 4"-radio knot, a "rather curious structure" described by Walker et al. (1987), which we interpret as the beam relaxation zone in the context of our two-flow model (Sol et al., 1989).

Benson, J.M. et al. (1988) Astrophys. J. 334, 560.

Pelletier, G., Sol, H., Asséo, E. (1988) Phys. Rev. A 38, 2552.

Sol, H., Pelletier, G., Asséo, E. (1989) MNRAS 237, 411.

Walker, R.C., Benson, J.M., Unwin, S.C. (1987) Astrophys. J. 316, 546.

Walker, R.C., Walker, M.A., Benson, J.M. (1988) Astrophys. J. 335, 668. 